\title{
A Retained Epidural Catheter Fragment Treated by Surgery
}

\author{
Kiyoshi Tarukado ${ }^{1}$, Takaaki Oda ${ }^{2}$, Osamu Tono ${ }^{1}$, Hiroyuki Suetsugu ${ }^{1}$, Toshio Doi ${ }^{1}$ \\ ${ }^{1}$ Department of Orthopedic Surgery, Kyushu University Beppu Hospital, Beppu, Japan \\ ${ }^{2}$ Department of Orthopedic Surgery, Takada Central Hospital, Bungotakada, Japan
}

The breakage of an epidural catheter is an extremely rare complication. We describe a unique case where a retained epidural catheter fragment after epidural anesthesia was treated by surgery. The epidural catheter broke during its removal, requiring surgery to remove the retained catheter. Intraoperatively, the removal of the catheter was attempted by simple traction, but was impossible because of the adhesion. The adhesion of the dura mater surface was carefully exfoliated and the successful removal of the catheter was accomplished. Conventionally, it was said that this follow-up was enough for the retained catheter. However, if a catheter is retained within the spinal canal, surgical removal should thus be considered before the adhesion advances.

Keywords: Epidural; Catheter; Complication; Surgery

\section{Introduction}

Epidural anesthesia is usually performed to provide intraoperative anesthesia, postoperative analgesia or cancer pain control using a catheter. Epidural anesthesia is also frequently used for alleviating labor pain and surgery involving the lower limbs in the field of orthopedics. Epidural catheters are ordinarily removed by physicians after the catheter is no longer needed for pain relief. The breakage of an epidural catheter is an extremely rare complication. Therefore, the management of retained fragments within the spinal canal remains controversial.

Herein we describe a unique case where a retained epidural catheter fragment was treated by surgery.

\section{Case Report}

An 82-year-old female with $60 \mathrm{~kg}$ body weight and 156 $\mathrm{cm}$ height who was otherwise healthy underwent a right total knee arthroplasty for osteoarthrosis under epidural anesthesia at a hospital nearby. The insertion of the epidural catheter and operation were finished without any problems and the removal of the epidural catheter was attempted as usual two days after the operation. The epidural catheter broke during its removal. The physician felt resistance when he tried to pull out the catheter and then the catheter was broken $13 \mathrm{~cm}$ from the tip. X-rays and a computed tomography scan were subsequently taken (Fig. 1). Although the catheter fragment retaining within the patient was long, a wait-and-see approach was initially employed because there was no neurological deficit. The effusion from the drain hole stopped two days later. No neurological deficit or fever developed after the first evaluation of the images. However, the migration of the catheter was found by imaging studies four weeks after the occurrence of the catheter breakage (Fig. 2). After detailed

Received Jun 9, 2014; Revised Jun 13, 2014; Accepted Jun 22, 2014

Corresponding author: Toshio Doi

Department of Orthopedic Surgery, Kyushu University Beppu Hospital,

4546 Tsurumihara Tsurumi, Beppu 874-0838, Japan

Tel: +81-977-27-1755, Fax: +81-977-27-1755, E-mail: toshidoi@ortho.med.kyushu-u.ac.jp 
discussions with the patient and her family it was decided that a surgical removal of the retained catheter should be performed. Then, the patient was referred to our hospital.

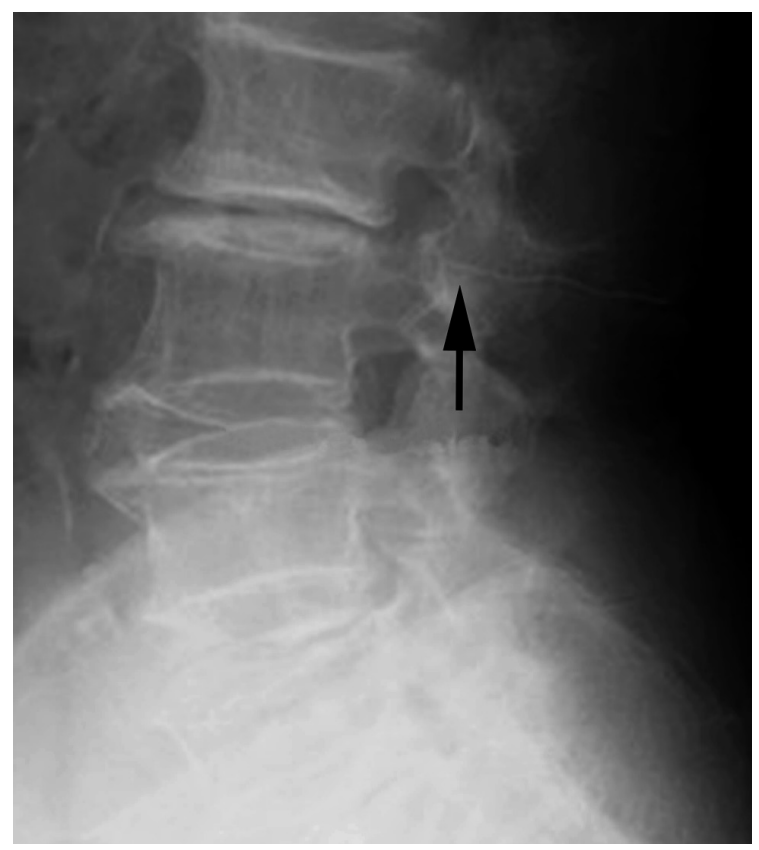

Fig. 1. Lateral X-ray taken just after the catheter breakage occurred. The arrow indicates the retained catheter.

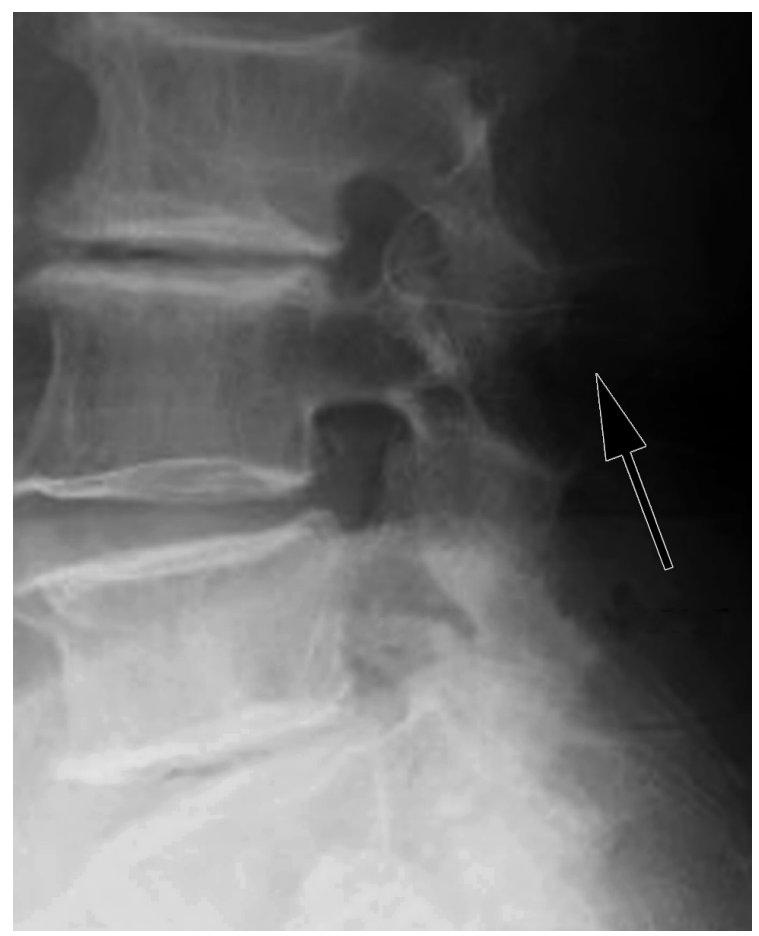

Fig. 2. Lateral X-ray taken four weeks after the catheter breakage occurred. The arrow indicates the migration of the retained catheter.
There was no neurological deficit at the time of admission. A laminectomy was performed at L2-3 seven weeks after the catheter had broken. The patient was placed in the prone position under general anesthesia and then the spinal level that needed to be treated and the retained catheter were identified by using an image intensifier. We exposed the spinal process at L2-3 and the L2 spinal process was split by an air drill. The catheter was confirmed to be present between the L2 and L3 spinous processes and the edge of the catheter was found to be broken (Fig. 3 ). The catheter went entered the epidural space through the flavum.

After removal of the bilateral flavum in an usual manner the catheter was found to be coiled up at the surface of the dura mater and was covered by a thin reactive film (Fig. 4). The catheter was heading to the nerve root. No evidence of dural puncture by the catheter was found. The removal of the catheter was first attempted by simple traction, however, was found to be impossible because of the adhesion (Fig. 5). The adhesion of the dura mater surface was carefully exfoliated and then the removal of the cath-

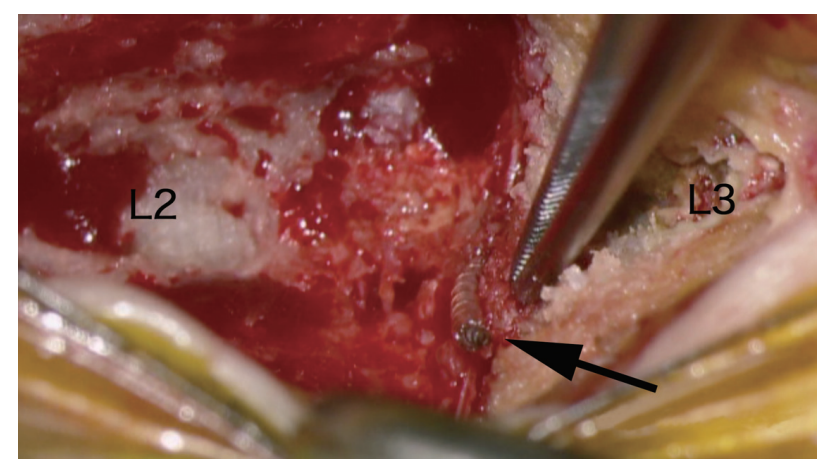

Fig. 3. The edge of the catheter (arrow) was found between the spinous process.

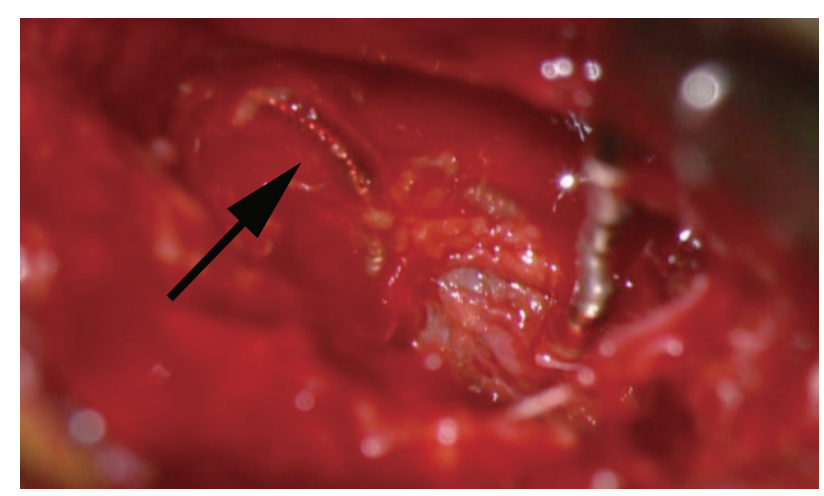

Fig. 4. The arrow indicates a thin reactive film that covered the retained catheter on the dura matter. 


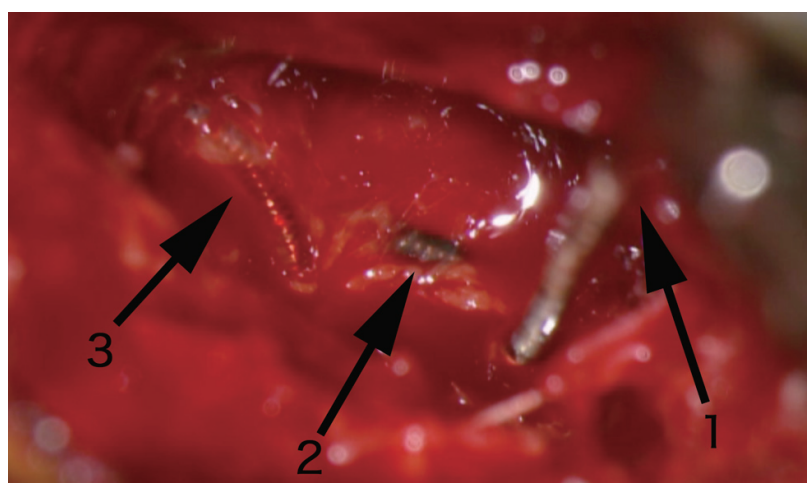

Fig. 5. The arrow (1) indicates the edge of the catheter that was withdrawn. The arrow (2) indicates the catheter movement was confirmed by traction. The arrow (3) indicates the catheter did not move by adhesion.

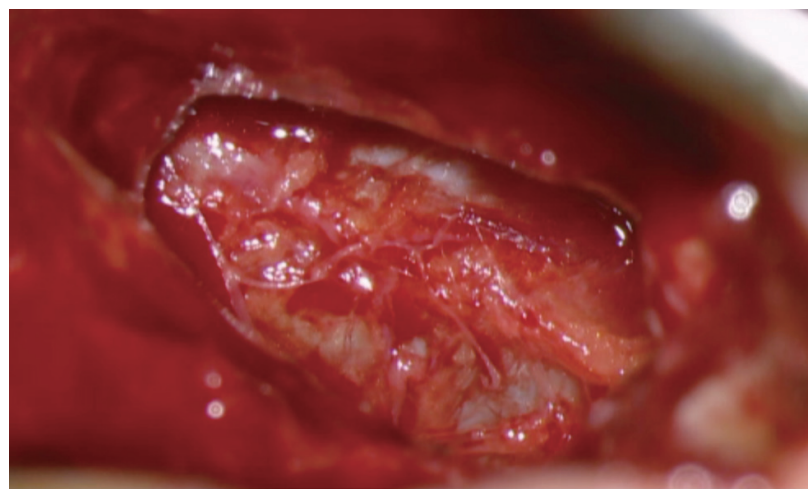

Fig. 6. Scar tissue was recognized on the surface of the dura mater after the catheter removal.

eter was accomplished (Fig. 6). No cerebral spinal fluid leakage was found after removal of the catheter.

\section{Discussion}

The causes of catheter breakage include various factors, such as characteristics of the catheter, poor technique during insertion or removal, catheter kinking or knotting and entrapment by the spinous process and interspinous ligaments or facets. In the present case, a reinforced catheter, which has some advantages compared to conventional non-reinforced catheters [1] was used and the insertion was performed in an usual manner. The catheter was inside made of stainless-steel coil body construction and outside of polyurethane. Although threading a catheter more than $5 \mathrm{~cm}$ has been reported to increase the risk of knotting [2], the catheter was inserted slightly longer in this case. Mitra and Fleischmann [3] reported several options for epidural catheter removal, however, the physician continued trying to remove the catheter despite encountering resistance in the present case.

The intraoperative findings showed catheter kinking on the surface of the dura mater, however, no catheter knotting was found. The retained catheter was worn out between the spinous process. This patient had a "kissing spine" where there was a close approximation of the spinous processes at adjacent levels. A "kissing spine" might be a risk factor for the shearing failure of catheters. In the present case, several problems, including poor technique, catheter kinking and entrapment by the spinous process appeared to be associated with the breakage of the catheter. If problems occur, it is important to follow the procedures suggested by Mitra et al. before applying excessive tension.

The management of a retained catheter within the spinal canal is controversial. Crawford [4] reported an incidence of catheter breakage of about $0.04 \%(12 / 27,000)$ in the 1980s. Recently, safer techniques have been widely accepted for the insertion or removal. The evolution of the catheters has been advancing too. Those factors helped to further decrease the incidences of catheter breakage. Collier [5] reported an incidence of catheter breakage of about $0.002 \%(1 / 60,000)$ in the 2000s.

No standard treatment for the management of a retained catheter within the spinal canal has established because it is an uncommon complication. One previous paper stated that "It is usually not necessary to remove a small amount of catheter in the epidural space. Thus, in general, laminectomy is reserved for situations associated with symptoms or signs" [6]. However, the breakage of an epidural catheter should be treated on a case-by-case basis. In the present case, the patient was elderly and so, non-surgical treatment was selected at first. The surgical removal was finally performed seven weeks after the catheter broke and adhesion was seen during the operation. Judging from the intraoperative findings of this case, when a foreign body exists in the spinal canal, it has a high potential to cause the development of granulation tissue. A large retained catheter within the spinal canal entails a great risk of extensive surgical invasion at some future point. Therefore, if the adhesion occurs between granulation tissue and the dura mater, the removal of the retained catheter wrapped with granulation tissue becomes difficult. Furthermore, once neurological sequelae develop, it is often difficult or impossible to reverse them. 
Consequently, if a portion of a catheter is retained within the spinal canal, surgical removal should be considered before the adhesion advances.

\section{Conflict of Interest}

No potential conflict of interest relevant to this article was reported.

\section{References}

1. Asai T, Yamamoto K, Hirose T, Taguchi H, Shingu K. Breakage of epidural catheters: a comparison of an arrow reinforced catheter and other nonreinforced catheters. Anesth Analg 2001;92:246-8.

2. Browne RA, Politi VL. Knotting of an epidural cath- eter: a case report. Can Anaesth Soc J 1979;26:142-4.

3. Mitra R, Fleischmann K. Management of the sheared epidural catheter: is surgical extraction really necessary? J Clin Anesth 2007;19:310-4.

4. Crawford JS. Some maternal complications of epidural analgesia for labour. Anaesthesia 1985;40:121925.

5. Collier C. Epidural catheter breakage: a possible mechanism. Int J Obstet Anesth 2000;9:87-93.

6. Veering BT, Cousins MJ. Epidural neural blockade. In: Cousins MJ, Carr DB, Horlocker TT, Bridenbaugh PO, editors. Cousins and Bridenbaugh's neural blockade in clinical anesthesia and management of pain. Philadelphia: Lippincott Williams \& Wilkins; 2009. p.241-95. 